Meta

Journal des traducteurs

Translators' Journal

\title{
La terminologie de l'entreprise
}

\section{Nada Kerpan}

Volume 20, numéro 1, mars 1975

Vingt ans de traduction. Bilan et prospectives

URI : https://id.erudit.org/iderudit/003310ar

DOI : https://doi.org/10.7202/003310ar

Aller au sommaire du numéro

Éditeur(s)

Les Presses de l'Université de Montréal

ISSN

0026-0452 (imprimé)

1492-1421 (numérique)

Découvrir la revue

Citer cet article

Kerpan, N. (1975). La terminologie de l'entreprise. Meta, 20(1), 71-74.

https://doi.org/10.7202/003310ar

Ce document est protégé par la loi sur le droit d'auteur. L'utilisation des services d'Érudit (y compris la reproduction) est assujettie à sa politique d'utilisation que vous pouvez consulter en ligne.

https://apropos.erudit.org/fr/usagers/politique-dutilisation/
Cet article est diffusé et préservé par Érudit.

Érudit est un consortium interuniversitaire sans but lucratif composé de l’Université de Montréal, l'Université Laval et l'Université du Québec à Montréal. Il a pour mission la promotion et la valorisation de la recherche. https://www.erudit.org/fr/ 


\section{La terminologie de l'entreprise}

\section{LA TERMINOLOGIE}

Dans tout pays traducteur, il se fait nécessairement de la terminologie et il en est de même de toute entreprise qui dispose d'un service de traduction. Toutefois, on semblait ignorer jusqu'à tout récemment, tant au Canada qu'au Québec et autant dans le secteur public que dans l'entreprise, que la terminologie existât ou dût exister en tant que telle, qu'elle fût pratiquée et qu'elle eût ses objectifs et ses prescriptions propres. Il est étonnant de constater que la classification type des professions du Bureau international du travail (édition 1968) ne signale point l'existence de terminologues, la terminologie étant souvent assimilée à la lexicographie $^{1}$ ou encore fortement encadrée par la traduction, voire même totalement amalgamée à cette dernière profession. Au pays, en fait, la terminologie n'a commencé à se structurer en activité autonome que voilà une quinzaine d'années ${ }^{2}$.

C'est en effet sous la poussée de la traduction ou du bilinguisme que se sont dégagés les besoins terminologiques et que, par voie de conséquence, s'est constituée une activité terminologique autonome. Au Bureau des traductions du Secrétariat d'État à Ottawa, la masse, la variété et la technicité des travaux ainsi qu'un objectif d'optimisation des services de traduction ont nécessité l'établissement d'un Centre de terminologie à vocation vaste et concrète. Au Québec, la primauté de l'anglais tant dans le domaine courant, scientifique ou technique que dans le secteur des affaires a amené l'Office de la langue française à concentrer, durant une certaine période, ses efforts sur la recherche terminologique. De son côté, la Banque de terminologie de l'Université de Montréal s'est consacrée - face aux impératifs internationaux, régionaux et locaux en matière linguistique - à la terminologie en tant que telle, la destinant à divers types d'usagers, dont l'entreprise privée.

\section{LA TERMINOLOGIE DANS L'ENTREPRISE}

Si la terminologie a pu s'établir en activité autonome dans le secteur public, tel n'est pas encore le cas dans l'entreprise privée. En effet, celle-ci commence à peine à se doter de services de traduction ou de services linguistiques. Des bureaux de traduction existent, il est vrai, depuis déjà 25 ans dans certaines grandes sociétés

1. Meta, VII, 6, juin 1974.

2. Paul A. Horguelin, Historique de la recherche et de la diffusion terminologiques au Canada ", Colloque de l'A.T.I.O. sur la terminologie appliquée à la traduction, Ottawa, septembre 1974. 
du Québec et, au fur et à mesure des travaux de traduction, il s'y exerçait une certaine activité terminologique, assurée par le traducteur même. Comme, dans la plupart des cas, le service de traduction était créé pour les besoins de la division des Relations publiques, il devient manifeste que la part faite à la recherche terminologique ne pouvait être que ténue. Les besoins en terminologie sont, il va de soi, réduits et de faible technicité lorsqu'il s'agit de correspondance, de publicité ou de communiqués à la presse, par exemple. Cependant, lorsque la traduction intéresse quelque domaine spécialisé ou technique, la terminologie constitue alors une fonction importante - sinon la fonction fondamentale - de l'activité traduisante ; de même, si l'entreprise opte pour la francisation du milieu du travail, elle pose la terminologie en élément essentiel de l'opération.

C'est ainsi que, dans les années 60 , quelques entreprises reconnaissent une certaine place à la terminologie, en déterminent l'importance et le fonctionnement. L'on songe en l'occurrence, par exemple, à l'Hydro-Québec qui se francise exclusivement; à Bell Canada qui établit un vaste programme de traduction de documents techniques et administratifs et officialise la terminologie française des postes cadres de l'entreprise ; au $\mathrm{CN}$ qui conçoit une structure linguistique complète pour les employés, dans laquelle la terminologie se présente en module distinct de la traduction et de l'enseignement des langues.

Toutefois, dans l'entreprise en général, la terminologie fait plutôt figure de parente pauvre de la traduction. On ne lui accorde que peu de temps dans la fébrilité de la traduction; on en laisse l'entière responsabilité au traducteur qui - faute de temps, de formation ou de documentation - la réduit trop souvent à une terminologie de traduction; comme toute recherche, elle paraît onéreuse et peu rentable ; et, de surcroît, elle réclame une documentation qui, ou bien, n'existe pas sous forme de dictionnaires bilingues, ou bien est difficile à obtenir de l'étranger sous forme de publications ou d'ouvrages de référence unilingues (à moins de missions documentaires ou terminologiques outre-Atlantique!), ou encore pour laquelle il n'a pas été prévu suffisamment de crédits. C'est donc dire que, même s'il semble aller de soi que la terminologie représente une partic essentielle du processus de traduction ou de francisation, il lui était refusé l'autonomie ou les moyens nécessaires soit à la réalisation d'une certaine qualité terminologique soit à la production terminologique même.

Aujourd'hui, dans les années 70 , la terminologie acquiert une importance de plus en plus grande dans l'entreprise. Le Centre de linguistique de l'entreprise (C.L.E.) conclut en effet, à la suite d'une enquête sur la situation de la traduction en 1974, que la terminologie est appelée à un rôle plus marqué dans le cadre des programmes de francisation ou de bilinguisation arrêtés par les compagnies. Toutefois, de signaler le rapport du C.L.E., seuls $25 \%$ des entreprises ayant un service de traduction disposent d'une section distincte de terminologie et la plupart de ces terminologues ne consacrent que 50\% de leur temps à leur activité propre, le reste étant employé à la traduction ou encorc à des fonctions administratives. En effet, la terminologie s'accomplit encore, en général, dans une structure mal adaptée tant aux besoins de la traduction et de la rédaction qu'à ses besoins particuliers. Aussi le rapport estime-t-il qu'il faudrait mieux organiser la recherche terminologique dans l'entreprise «afin de rendre plus efficace cet aspect de la traduction». 
En fait, l'entreprise reconnaît de plus en plus la nécessité de travaux terminologiques suivis et c'est pourquoi elle accorde à ses traducteurs le droit d'effectuer de la recherche et de confectionner des fiches, selon un protocole établi ou non, ou encore affecte-t-elle temporairement - faute d'unité terminologique ou par insuffisance de personnel à cette section - un traducteur à des recherches données. $\mathrm{Ce}$ qui caractérise l'entreprise, c'est que sa production terminologique a été, jusqu'à présent, le produit de traducteurs habitués aux rouages de la compagnie. De plus, presque tous les terminologues actuels d'entreprise sont d'anciens employés du service même de traduction. Est-ce à dire que la terminologie d'entreprise ne pourra jamais être effectuée que par des traducteurs d'expérience consacrés, ou non, terminologues? À vrai dire, la terminologic d'entreprise présente des servitudes telles, de tels aspects et particularités, et des fonctions si diverses que, à l'heure actuelle, c'est le traducteur pourvu d'aptitudes pour la recherche et comptant une certaine expérience à la compagnie qui est le mieux désigné pour assurer les services terminologiques nécessaires à l'équipe de traduction, aux rédacteurs et aux employés de l'entreprise.

En effet, la terminologie pratiquée dans l'entreprise se distingue sous bien des rapports de celle qui s'effectue à l'Office de la langue française, à la Banque de terminologie de l'Université de Montréal, voire même au Secrétariat d'État. Elle n'y jouit pas et jamais elle n'y jouira d'une totale autonomie puisqu'elle sera, longtemps encore, étroitement liée à la traduction; qu'elle est essentiellement une terminologie de grande consommation et même de consommation immédiate étant au service tant du traducteur, du rédacteur et de l'enseignant d'entreprise que de l'employé des services opérationnels et fonctionnels ; qu'elle est fortement soumise au paramètre «temps »; qu'elle ne peut se faire en vase clos, mais plutôt en étroite collaboration avec les spécialistes de la technique ou du domaine en cause ; et qu'elle est souvent contestée par un personnel généralement très anglicisé ou encore en proie au complexe ou à la crainte du « français de France ».

\section{LE TERMINOLOGUE D'ENTREPRISE}

Si le terminologue d'entreprise doit avoir la rigueur intellectuelle du chercheur de la Banque de terminologie, de l'Office de la langue française ou du Secrétariat d'État, s'il lui faut établir des fiches valables et préparer des listes lexicales ou lexiques complexes, ses responsabilités ne se limitent pas à cette seule activité. Il doit à la fois se doubler d'un documentaliste, d'un linguiste, d'un agent de relations publiques, et parfois même d'un administrateur.

Souvent seuls ou très peu nombreux, comptant sur un personnel de soutien réduit ou peu formé, les terminologues d'entreprise ont la charge de constituer le centre de documentation même. C'est à eux qu'incombe la tâche d'établir une docurnentation générale et technique qui réponde aux exigences courantes et futures, de consulter les ouvrages bibliographiques, de sélectionner les volumes et publications nécessaires, d'effectuer des missions documentaires dans d'autres entreprises ou à l'étranger ; bref, de mettre sur pied une «bibliothèque » adaptée aux besoins et des traducteurs et des autres employés de la compagnie, d'en assurer la mise à jour, et de déterminer les moyens d'accès à la documentation. 
Le terminologue d'entreprise doit également fournir des services de consultation linguistique, qu'il s'agisse de grammaire, de stylistique, d'orthographe, de lexicographie, de traduction ou de terminologie en tant que telle. Non seulement est-il à la disposition du personnel de la traduction, mais encore du rédacteur au service des Relations publiques ou de l'employé improvisé, pour les besoins, rédacteur ou traducteur, du personnel opérationnel, du professeur qui désire de la terminologie spécialisée pour un enseignement pratique des langues du travail, et du public.

Il doit, en outre, assurer des liaisons efficaces tant au niveau du service de traduction qui a recours à lui, et dont il coordonne ou dirige l'activité terminologique, qu'avec les autres services de l'entreprise dont il doit déterminer les besoins en matière de terminologie. Il doit aussi entretenir des rapports étroits avec les spécialistes de l'entreprise ou des entreprises connexes, avec les terminologues de l'extérieur, avec l'Office de la langue française, avec la Banque de terminologie de l'Université de Montréal et avec l'étranger.

S'il est responsable d'une équipe de terminologie, il devient alors administrateur de personnel ainsi que des crédits du centre de documentation.

Et il doit être en même temps, et avant tout, terminologue - c'est-à-dire analyser, évaluer et dépouiller la documentation regroupée sur un sujet donné; confectionner les fiches et les lexiques nécessaires; effectuer des études terminologiques ; publier et diffuser les travaux terminologiques réalisés. Il a, de plus, pour mission de normaliser la terminologie utilisée dans l'entreprise; de former des commissions terminologiques au sein de la compagnie ou avec d'autres entreprises, commissions constituées de personnel linguistique mais aussi de spécialistes du domaine à l'étude. En effet, contrairement à son homologue de la Banque de terminologie ou encore de l'Office, le terminologue d'entreprise ne peut faire abstraction de l'utilisateur de la technique ou de la langue. Celui-ci, comme on le signalait précédemment, revendique ou encore s'arroge le droit d'être consulté puisqu'en définitive, comme il l'estime, il sera l'usager du terme et qu'il n'entend pas être gêné par des termes ultrasavants ou abscons. Aussi le terminologue d'entreprise doit-il être en quelque sorte un agent de commercialisation du français (le plus souvent), faire tomber les réserves injustifiées de l'employé pour l'amener à utiliser une terminologie correcte, et en faire un organe de diffusion efficace et rapide.

Enfin, le terminologue d'entreprise est en général pressé par une demande extrêmement variée et par le temps. C'est pourquoi il doit produire une réponse qui soit la plus juste possible, la plus définitive possible et faite dans les plus brefs délais. Son rôle, à l'heure actuelle, se diversifie en une multitude d'actions et de fonctions qui, vues sous un rapport, confèrent à la terminologie d'entreprise une efficacité et un dynamisme certains. Toutefois, considérés sous l'angle exclusif de la profession terminologique, elles invitent plutôt à une restructuration qui déchargera le personnel de certaines tâches secondaires ou accessoires' et lui laissera une plus grande disponibilité pour la recherche et les travaux terminologiques en tant que tels.

NADA KERPAN 